$\mathrm{UW} / \mathrm{PT}$ 00-01

\title{
Sphaleron rate in the symmetric electroweak phase
}

\author{
Guy D. Moore \\ Department of Physics, University of Washington, Seattle WA 98195-1560 USA
}

(January 2000)

\begin{abstract}
Recently Bödeker has presented an effective infrared theory for the dynamics of Yang-Mills theory, suitable for studying the rate of baryon number violation in the early universe. We extend his theory to include Higgs fields, and study how much the Higgs affects the baryon number violation rate in the symmetric phase, at the phase coexistence temperature of a first order electroweak phase transition. The rate is about $20 \%$ smaller than in pure Yang-Mills theory. We also analyze the sphaleron rate in the analytic crossover regime. Our treatment relies on the ergodicity conjecture for 3-D scalar $\phi^{4}$ theory.
\end{abstract}

\section{INTRODUCTION}

Baryon number is violated in the standard model [1], and while the rate of the violation is negligibly small in vacuum, it can be significant at higher temperatures [2, 3].

Recently there has been substantial interest in how fast baryon number is violated in the standard model at high temperatures. This is both because the rate is important for baryogenesis, and because it is an interesting problem in the more general framework of understanding dynamics of hot gauge theories.

The last 3 years have seen major progress in this problem. First, analytic work has clarified what physics is relevant. While it has long been known that infrared or "soft" gauge fields with momenta $p \sim g^{2} T$ are responsible for baryon number violation [3], the recent work of Arnold, Son, and Yaffe has shown that "hard" modes with momenta $p \sim T$ play an essential role in modifying the dynamics of the $p \sim g^{2} T$ degrees of freedom [ [4] [ Bödeker has gone further, showing the role of scatterings between such hard modes by exchange of $g^{2} T \lesssim p \lesssim g T$ modes [7] 9 . There has also been progress in numerically studying the effective infrared (IR) theories to determine the size of baryon number violation [10,11].

The rate of baryon number violation is characterized by the sphaleron rate]

$$
\begin{aligned}
\Gamma & \equiv \lim _{V, t \rightarrow \infty} \frac{\left\langle\left(N_{\mathrm{CS}}(t)-N_{\mathrm{CS}}(0)\right)^{2}\right\rangle}{V t}, \\
N_{\mathrm{CS}}(t)-N_{\mathrm{CS}}(0) & =\frac{1}{16 \pi^{2}} \int_{0}^{t} \int d^{3} x \epsilon_{i j k} E_{i}^{a} F_{j k}^{a} .
\end{aligned}
$$

\footnotetext{
${ }^{1}$ Our normalization is $D_{i}=\partial_{i}+i T^{a} A_{i}^{a}$, and $F_{i j}=\left[D_{i}, D_{j}\right] . \alpha$ means $\alpha_{w}$, and $g$ means $g_{w}$.
} 
Bödeker has shown that in pure Yang-Mills theory, $\Gamma$ has the parametric form [7]

$$
\Gamma=\kappa^{\prime}\left(\log \frac{m_{\mathrm{D}}}{g^{2} T}+C+O(1 / \log )\right)\left(\frac{g^{2} T^{2}}{m_{\mathrm{D}}^{2}}\right) \alpha^{5} T^{4} .
$$

The constant $C$ can and recently has been determined analytically [12]. The leading coefficient $\kappa^{\prime}$ can be obtained numerically by studying a local, UV finite theory, which is precisely Langevin dynamics for classical 3 dimensional nonabelian gauge fields:

$$
\begin{aligned}
D_{\tau} A_{i}^{a}(x, \tau) & =-g^{2} \frac{\partial H_{A}(A(\tau))}{\partial A_{i}^{a}(x, \tau)}+\xi_{i}^{a}(x, \tau) \\
H_{A} & =\int d^{3} x \frac{1}{4 g^{2}} F_{i j}^{a} F_{i j}^{a}(x), \quad-g^{2} \frac{\partial H_{A}}{\partial A_{i}^{a}}=\left(D_{j} F_{j i}\right)^{a}=(D \times B)_{i}^{a}, \\
\left\langle\xi_{i}^{a}\left(x_{1}, \tau_{1}\right) \xi_{j}^{b}\left(x_{2}, \tau_{2}\right)\right\rangle & =2 g^{2} T \delta\left(x_{1}-x_{2}\right) \delta\left(\tau_{1}-\tau_{2}\right) \delta_{i j} \delta^{a b},
\end{aligned}
$$

Here $D_{\tau}$ is the covariant derivative in Langevin time $\tau, F_{i j}^{a}$ the (nonabelian) magnetic field, and $\xi$ Gaussian white noise normalized as shown. f $^{\text {. }}$

The form of this theory is identical to the nonabelian Ampere's law, but with the current replaced with $\vec{j}=\sigma \vec{E}$, as we would expect in a conducting medium;

$$
D \times B=-\sigma E(+ \text { noise }),
$$

with the Langevin time identified as $\tau=\sigma t$. Here $\sigma$ is a "color conductivity" which describes the current response to very infrared external fields. It can be treated as a constant, to (next to) leading order in $\log (1 / g)$, because the mean free path for color changing collisions is shorter than the $1 / g^{2} T$ scale where $\Gamma$ is set, by a factor of $\log (1 / g)$. An explicit expression for $\sigma$ at next to leading log order, found by Arnold and Yaffe, [13], will be presented in Eq. (3.23). $\kappa^{\prime}$ is determined in this theory as

$$
\kappa^{\prime}=\frac{3 \sigma}{2 \pi} \lim _{V, \tau \rightarrow \infty} \frac{\left\langle\left(\left(N_{\mathrm{CS}}(\tau)-N_{\mathrm{CS}}(0)\right)^{2}\right\rangle\right.}{V \tau} .
$$

This effective theory was studied numerically in [14], with the result (see below) that $\kappa^{\prime} \simeq 10$. This effective theory is not sufficient to determine the $O(1 / \log )$ coefficient in Eq. (1.3), and neither will anything we discuss.

Most of what we have described, and in particular everything in references [4, [7, [2, 14, 15], is for the case of pure Yang-Mills theory. This is appropriate if we are interested in temperatures very much higher than the electroweak phase transition (or crossover) temperature, because in that case the Higgs boson has a large thermal mass and can be removed perturbatively. However, the main application we are interested in, baryogenesis, requires knowing $\Gamma$ in a theory with at least one Higgs boson, in the symmetric phase but at or slightly below the equilibrium temperature $T_{\text {eq }}$ for the electroweak phase transition. It is not clear

\footnotetext{
${ }^{2}$ Actually it is not a constant, but contains a $\log \left(\log \left(m_{D} / g^{2} T\right)\right)$, see Eq. (3.23).

${ }^{3}$ Note that the Langevin time $\tau$ has dimensions of length squared, and recall our scaling convention for $A$.
} 
whether discarding the Higgs physics is justified in this case, and we should rethink both the appropriate effective theory, and the numerical determination of $\Gamma$, in this light.

In this paper we will attempt to fill this gap. First we discuss how important we expect Higgs physics to be by considering the thermodynamics of Yang-Mills Higgs fields in Section III. Then we construct an appropriate infrared (IR) effective theory which generalizes Bödeker's effective theory in Section III. We discuss the numerical implementation in Section $\Pi \nabla$ and present numerical results in $\nabla$. We find that, where the phase transition is strong enough to preserve baryon number after its completion, the change in $\Gamma$ due to Higgs physics is quite a small effect, so the error in using pure Yang-Mills theory is small, of order $20 \%$. While we work in the minimal standard model (at experimentally excluded values of the Higgs mass, to get a strong enough phase transition), we expect the results to hold as well in extensions such as the MSSM with a light scalar top quark. This conjecture could be tested by simulations in that theory, along the lines of what we do here.

\section{THERMODYNAMIC INFLUENCE OF THE HIGGS}

To get an idea of how important Higgs physics will be for the sphaleron rate, we will try to get an idea of how important it is thermodynamically for the infrared transverse gauge boson excitations which we expect to be responsible for baryon number violation. As we will see, this in fact gives a reasonable estimate for what difference the Higgs will make in the sphaleron rate.

To a very good approximation the thermodynamics of infrared bosonic fields in the hot electroweak theory can be described by a three dimensional path integral [16]. In fact this can be understood as a special case of the statement that the IR physics is essentially classical, since the three dimensional path integral we arrive at coincides with the partition function of the classical bosonic theory. Up to parametrically suppressed correctionst the partition function governing the thermodynamics is [16]

$$
\begin{aligned}
Z=\int \mathcal{D} A_{i} \mathcal{D} A_{0} \mathcal{D} \Phi \exp -H / T \\
H=\int d^{3} x \frac{1}{4 g^{2}} F_{i j}^{a} F_{i j}^{a}+\frac{1}{2 g^{2}}\left(D_{i} A_{0}\right)^{a}\left(D_{i} A_{0}\right)^{a}+\frac{m_{\mathrm{D}}^{2}(T)}{2 g^{2}} A_{0}^{a} A_{0}^{a}+\frac{\lambda_{A}}{4 g^{4}}\left(A_{0}^{a} A_{0}^{a}\right)^{2} \\
\quad+\left(D_{i} \Phi\right)^{\dagger}\left(D_{i} \Phi\right)+m_{H}^{2}(T) \Phi^{\dagger} \Phi+\lambda\left(\Phi^{\dagger} \Phi\right)^{2}+\frac{\lambda_{A \Phi}}{2 g^{2}} A_{0}^{a} A_{0}^{a} \Phi^{\dagger} \Phi
\end{aligned}
$$

The couplings $\lambda, g^{2}, \lambda_{A} \sim \alpha^{2}$, and $\lambda_{A \Phi}=g^{2} / 2+O\left(\alpha^{2}\right)$ are determined by a matching calculation; $g^{2}, \lambda$, and the field wave function normalizations correspond to those of the 4-D theory at a renormalization point given roughly by $T$. We will always treat $\lambda \sim g^{2}$ for power counting purposes, as is appropriate given the renormalization structure of the theory. It is

\footnotetext{
${ }^{4}$ The 3-D theory should be viewed as an IR effective theory for the thermodynamics below the scale $T$. At some level of precision it becomes necessary to include high dimension operators. If we are interested in thermodynamics at the length scale $1 / g^{2} T$, then neglecting the high dimension operators causes errors of $O\left(\left(g^{2} T / T\right)^{2}\right)$ times an additional explicit factor of $\alpha$ because the high dimension operators are radiatively induced, leading to an $\alpha^{3}$ error. Of course, on less infrared scales the effective theory is less accurate.
} 


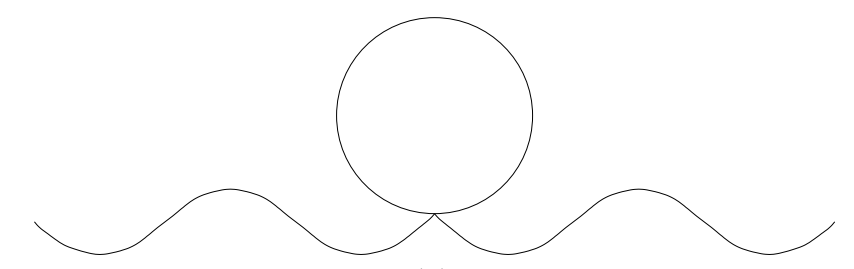

(a)

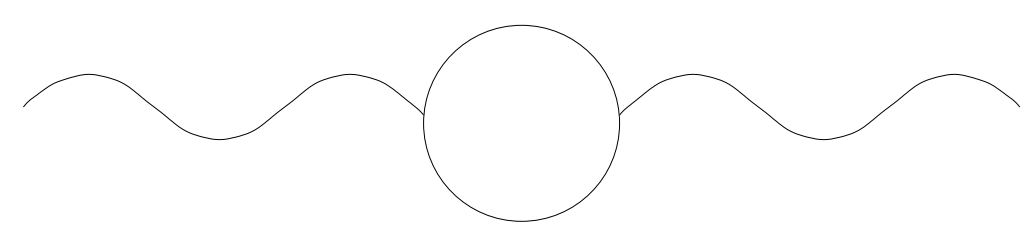

(b)

FIG. 1. gauge field self-energy insertions generated by the Higgs fields.

sometimes useful to consider $\lambda \ll g^{2}$ and to expand in $\lambda / g^{2}$, but if we do so it is implied that $\lambda$ is still $\gg g^{4}$, for instance. $A_{0}$ is the remnant of the temporal connection and can be thought of as an adjoint scalar field. Its mass term is the Debye mass responsible for charge screening, and is given by

$$
m_{\mathrm{D}}^{2}(T)=\frac{g^{2} T^{2}}{12}\left(4 N_{\mathrm{c}}+N_{f}+2 N_{s}\right)-3 \mathrm{D} \text { counterterm }+O\left(g^{4} T^{2}\right),
$$

with $N_{\mathrm{c}}=2$ the second Casimir of the group, $N_{f}$ the number of chiral, fundamental representation fermions, and $N_{s}$ the number of fundamental representation complex scalars. In the minimal standard model, the $O\left(g^{2}\right)$ piece is $m_{\mathrm{D}}^{2}=(11 / 6) g^{2} T^{2}$.

Because of the Debye mass, the $A_{0}$ field is heavy and has little influence on the very infrared thermodynamics, leading only to a rescaling of the effective gauge coupling by an $O(g)$ correction [16]. However the same is not generically true for the Higgs field. This is because, unlike the $A_{0}$ field, it has a negative vacuum mass squared which may approximately cancel the positive induced thermal mass;

$$
\begin{aligned}
& m_{H}^{2}(T)=m_{\mathrm{th}}^{2}+m_{\mathrm{vac}}^{2}, \quad m_{\mathrm{vac}}^{2}<0, \quad m_{\mathrm{th}}^{2} \sim g^{2} T^{2}, \\
& \left(\text { specifically, } m_{\mathrm{th}}^{2}=\frac{\left(3 g^{2}+g^{\prime 2}+4 y_{t}^{2}+8 \lambda\right) T^{2}}{16}\right. \text { in the MSM). }
\end{aligned}
$$

At very large temperatures, $m_{H}^{2}(T) \sim g^{2} T^{2}$ and its thermodynamic effects are parametrically suppressed. However, the electroweak phase transition occurs where the positive thermal mass squared and the negative tree one cancel up to $O\left(g^{4} T^{2}\right)$ corrections, and it is in this regime that we need to know the sphaleron rate. It makes sense, then, to treat $m_{H}^{2}(T)$ parametrically as $O\left(g^{4} T^{2}\right)$, though depending on the strength of the phase transition, it may either be "large" or "small" within this parametric range.

Let us now consider the thermodynamic influence of the Higgs boson on the YangMills fields. Since we are only making estimates here, we will be content with one loop calculations even where the loop expansion is not very reliable. We can estimate the Higgs field's importance by considering Higgs contributions to the gauge field self-energy at soft external momentum $p \sim g^{2} T$. The sum of diagram (a) in Fig. 1 and any $p$ independent 
contributions from (b) vanish in any gauge invariance respecting regularization; the scalar contribution to the gauge field self-energy is then

$$
\Pi_{i j}^{a b}(p)=\frac{g^{2} T \delta^{a b}}{2} \int \frac{d^{3} k}{(2 \pi)^{3}}\left(\frac{2 k_{i} 2 k_{j}}{\left(\left(k-\frac{p}{2}\right)^{2}+m_{H}^{2}\right)\left(\left(k+\frac{p}{2}\right)^{2}+m_{H}^{2}\right)}-\frac{2 k_{i} 2 k_{j}}{\left(k^{2}+m_{H}^{2}\right)^{2}}\right) .
$$

If we take $p \ll m_{H}$, the result is

$$
\Pi_{i j}^{a b}(p)=-\frac{g^{2} T \delta^{a b}}{48 \pi m_{H}}\left(\delta_{i j} p^{2}-p_{i} p_{j}\right)
$$

whereas in the regime where $m_{H} \ll p$, we get

$$
\Pi_{i j}^{a b}(p)=-\frac{g^{2} T \delta^{a b}}{32|p|}\left(\delta_{i j} p^{2}-p_{i} p_{j}\right)
$$

In either case the sign of the effect is such that it reduces the size of infrared gauge field excitations; so the presence of a Higgs field should reduce $\Gamma$.

The calculation is for the symmetric phase, where there is no Higgs condensate. When there is a condensate $\phi_{0}$ (normalized as $\phi_{0}^{2}=\left\langle 2 \Phi^{\dagger} \Phi\right\rangle$ ) then there is an induced mass for the gauge bosons of $m_{W}^{2}=g^{2} \phi_{0}^{2} / 4$, which will of course further reduce $\Gamma$. However, we are primarily interested right now in the symmetric phase case.

To decide how important the scalar contribution is, we need to know what momentum $p$ is characteristic for the baryon number violating processes we intend to study. Since the physics we are after is nonperturbative physics, we expect the answer to be, $p$ such that perturbation theory is breaking down. We can estimate what $p$ is necessary by computing the contribution of gauge bosons to the self-energy. The result is not gauge invariant, but in Landau gauge the analogous contribution from gauge and ghost loops is 0

$$
\Pi_{i j}^{a b}(p) \text { from bosons }=\frac{11 N_{\mathrm{c}} g^{2} T \delta^{a b}}{64|p|}\left(\delta_{i j} p^{2}-p_{i} p_{j}\right) .
$$

The sign is the opposite, indicating that gauge self-interactions lead to a more rapid onset of nonperturbatively large fields. The loop contribution comes on order the tree inverse propagator $p^{2} \delta_{i j}-p_{i} p_{j}$, and the calculation therefore breaks down completely, for $p \sim g^{2} T / 3$. We take this as a fair estimate of the nonperturbative scale, though such an estimate certainly cannot be considered accurate to better than about a factor of 2 .

For a heavy Higgs boson, then, we expect a correction of order $\left(g^{2} T / m_{H}(T)\right) /(48 \pi)$; but even for $m_{H}(T) \simeq 0$ we only expect a correction from the Higgs boson to the propagator at the relevant momentum to be of order $1 / 11$. This corresponds to a rescaling by $\sim(1 / 11)$ of the length scale where perturbation theory breaks down. However, since the fifth power of this length scale enters the sphaleron rate, the importance of the Higgs boson would be a little larger, as large as a $(10 / 11)^{5} \simeq 0.6$ reduction of $\Gamma$. This analysis also implies that,

\footnotetext{
${ }^{5}$ In Feynman gauge the 11 becomes 14. The answer differs from that in 17, for instance, because we have left out the (heavy) $A_{0}$ field, treated as light there.
} 
when there is a condensate, it starts to significantly suppress baryon number violation when the induced gauge field mass is of order $g^{2} T / 3$, which requires $\phi_{0} \simeq 2 g T / 3$. Of course for such a small condensate, the perturbative notion of "condensate" is lost and what we are doing is unreliable; but this should give us some estimate of how strong the transition needs to be before the drop in the sphaleron rate becomes significant.

In the standard model and in the regime where the phase transition is strong, we can estimate the symmetric phase $m_{H}\left(T_{\text {eq }}\right)$; at leading order in $\lambda / g^{2}$, a standard calculation from the curvature of the one loop effective potential gives

$$
m_{H}\left(T_{\mathrm{eq}}\right)=g^{2} T /\left(16 \pi \sqrt{\lambda / 2 g^{2}}\right) .
$$

Plugging this into Eq. (2.6) gives a self-energy correction of $-\sqrt{\lambda / 18 g^{2}}$, and a correction to the sphaleron rate of

$$
\frac{\delta \Gamma}{\Gamma}=-5 \sqrt{\frac{\lambda}{18 g^{2}}} .
$$

Below we will study the case where $\lambda / g^{2}=0.036$, for which the correction is $22 \%$. However, this mass is not in the regime $m_{H} \gg p$ for $p=g^{2} T / 3$, so we have computed Eq. (2.5) numerically for this $m_{H}$ and $p$; it yields -0.037. A rough estimate is that we will see $\Gamma$ reduced to $(1-.037)^{5}=.83$ of the Yang-Mills theory value. This estimate is quite rough, but it gives the idea that the symmetric phase rate will be lower, but not much lower, than the pure Yang-Mills theory rate.

To conclude, at the thermodynamic level, one would expect the Higgs field induced correction to infrared gauge field behavior, and hence to the sphaleron rate, to be rather small in the symmetric phase, and of order 1 for condensates smaller than $\phi_{0} \simeq(2 / 3) g T$. Naturally, for larger condensates the suppression becomes very substantial, see [3, 18].

\section{EFFECTIVE THEORY IN THE PRESENCE OF THE HIGGS}

Now we will go about constructing appropriate effective theories for studying baryon number violation when there is a light Higgs boson. Our treatment will follow very closely that of [0,7,4. Indeed, most of the complications stem from the Yang-Mills sector and have been resolved in the literature cited; the Higgs boson will introduce comparatively minor new complications.

We will also leave out the $U(1)$ field in what follows, even though it is light. This is a common and probably reasonable approximation. We make it partly because we expect the influence of the $\mathrm{U}(1)$ field to be weak; there is no direct interaction between the $\mathrm{SU}(2)$ and $\mathrm{U}(1)$ gauge fields, and the U(1) physics makes rather small modifications to the thermodynamics of the $\mathrm{SU}(2)$-Higgs system [19]. Also, including the $\mathrm{U}(1)$ physics would prove to be a significant complication, since at the $k \sim g^{2} T$ scale the U(1) fields are overdamped but with a $k$ dependent damping which must be treated as a nonlocal effect even in the final effective theory. We will not try to address this problem here.

Since we are not concerned with cases where there is a very large condensate $\phi_{0} \sim T / g$, degrees of freedom with momentum $k \sim T$ behave at leading order as massless free fields 
propagating in the background of the IR fields, and the degrees of freedom with $k \leq g T$ have large occupation numbers and can be treated as classical fields. The infrared behavior of the theory is described by a classical effective theory in which the $k \sim T$ degrees of freedom have been analytically integrated out. The resulting equations of motion are similar to those of the classical field theory. Defining

$$
\begin{aligned}
H & =H_{A}+H_{\Phi}+H_{E}+H_{\Pi}, \\
H_{A} & =\int d^{3} x \frac{1}{4 g^{2}} F_{i j}^{a} F_{i j}^{a}(x), \quad F_{i j} \equiv\left[D_{i}, D_{j}\right], \\
H_{\Phi} & =\int d^{3} x\left(D_{i} \Phi\right)^{\dagger}\left(D_{i} \Phi\right)(x)+m_{H}^{2} \Phi^{\dagger} \Phi(x)+\lambda\left(\Phi^{\dagger} \Phi\right)^{2}(x), \\
H_{E} & =\int d^{3} x \frac{1}{2 g^{2}} E_{i}^{a} E_{i}^{a}(x), \quad E_{i} \equiv\left[D_{t}, D_{i}\right], \\
H_{\Pi} & =\int d^{3} x \frac{1}{2} \Pi^{\dagger} \Pi(x), \quad \Pi \equiv D_{t} \Phi,
\end{aligned}
$$

the effective field equations will be the classical equations of motion derived from this Hamiltonian, supplemented by the hard thermal loop effects which arise from the integration over the heavy modes. The hard thermal loop (HTL) field equations are 20 23]

$$
\begin{aligned}
\left(D_{t} E_{i}\right)^{a}(x) & =-g^{2} \frac{\partial H_{A}}{\partial A_{i}^{a}(x)}-g^{2} \frac{\partial H_{\Phi}}{\partial A_{i}^{a}(x)}-m_{\mathrm{D}} \int \frac{d \Omega_{v}}{4 \pi} v_{i} W^{a}(x, v), \\
D_{t} \Pi(x) & =-\frac{\partial H_{\Phi}}{\partial \Phi^{\dagger}(x)}-m_{\mathrm{th}}^{2} \Phi(x) \\
& =D_{i} D_{i} \Phi(x)-\left(m_{H}^{2}(T)-2 \lambda \Phi^{\dagger} \Phi(x)\right) \Phi(x), \\
\left(D_{t} W\right)^{a}(x, v) & =-v_{i}\left(D_{i} W\right)^{a}(x, v)+m_{\mathrm{D}} v_{i} E_{i}^{a}(x), \\
\left(D_{i} E_{i}\right)^{a}(x) & =g^{2}\left(\Pi^{\dagger} i T^{a} \Phi(x)+\text { c.c. }\right)+m_{\mathrm{D}} \int \frac{d \Omega_{v}}{4 \pi} W^{a}(x, v) .
\end{aligned}
$$

These equations determine the field evolution, given initial information for $A, E$, $\Phi, \Pi$, and $W$, up to the freedom to choose the time dependent gauge. The last equation is Gauss' Law and needs to be applied as a constraint on the initial conditions; it commutes with the other equations so it remains valid at later times.

The only hard thermal loop effect on the soft scalar field is a thermal mass squared correction. We discuss this point at some length in Appendix A, where we demonstrate the absence of all other HTL effects involving scalar external lines. We write the correction as $m_{\mathrm{th}}^{2}$ (the thermally induced mass squared) and the sum $m_{H}^{2}+m_{\mathrm{th}}^{2}=m_{H}^{2}(T)$. In what follows we will absorb this shift in $m_{H}^{2}$ into $H_{\Phi}$. This does not require any modification of Eq. (3.6) because $m_{\mathrm{th}}^{2} \Phi^{\dagger} \Phi$ is independent of $A$.

The HTLs for gauge fields are much more complicated, and are implemented here with auxiliary fields, the $W$ fields, which allow them to be written in a local way [22,23]. The penalty is that the $W$ fields depend on direction $v$ as well as position $x$. Here $v$ is a unit vector, and $d \Omega_{v}$ is an integral over directions (the unit sphere) normalized so $\int d \Omega_{v} / 4 \pi=1$. We normalize $W$ slightly differently than the references, absorbing a factor of $m_{D}$ into its normalization so it enters the Hamiltonian with the same weight as the gauge fields. Its 
listing in Eq. (3.1) would be

$$
H_{W}=\int d^{3} x \frac{1}{2 g^{2}} \int \frac{d \Omega_{v}}{4 \pi} W^{a}(x, v) W^{a}(x, v)
$$

It is possible to derive the field equations from this (generalized) Hamiltonian, together with Eq. (3.1), but it requires rather nontrivial Lie-Poisson brackets [24. Note that the hard scalar contributions to the gauge HTL is identical in structure to that from hard gauge and fermionic degrees of freedom. This is most easily seen in the kinetic theory derivation of the gauge HTL's [22], where one finds that the spin of a hard degree of freedom only matters at subleading order in $g$.

Eqs. (3.6)-(3.9) already give an effective theory which is amenable to numerical treatment along the lines of that given for pure Yang-Mills theory in [10,11]. The added complication of including the Higgs field is much less than that involved in treating the hard thermal loops. However, such a numerical implementation is not ideal, because the system of equations presented is not UV finite. In particular the classical gauge and scalar degrees of freedom, with dynamics determined by Eqs. (3.6) and (3.7), generate UV divergent loop corrections which can be considered as extra contributions to the hard thermal loops. In a lattice regularization, these extra contributions are finite but grow linearly as the lattice spacing is made smaller, and are not rotationally invariant [25]. So long as $m_{D}$ is kept suitably large this should be a subdominant effect, but it is not clear that it is safe to make $m_{D}$ as large as the inverse lattice spacing. Because of these problems it is not easy to extract high precision information from such a simulation, and if we expect a fairly small change to $\Gamma$ due to the Higgs bosons, it may be problematic to isolate it from lattice spacing effects. Rather, we will follow Bödeker [0] and integrate out more degrees of freedom to construct what ultimately proves to be a simpler and cleaner effective theory (though no longer valid to corrections parametrically suppressed by a full power of $g$ ), which will prove a better test-bed for studying the importance of Higgs physics.

With this in mind we consider integrating over the $g T$ scale, down to some intermediate scale $\mu \ll g T$. We are also removing physics with a frequency scale $\omega \sim g T$; however we cannot directly consider only degrees of freedom with $\omega \ll k \ll g T$; for now $\omega$ is permitted to be as large as $\mu$.

Here we will only treat the case in which there is not a Higgs boson condensate of order $\phi_{0} \sim \mu / g$. This either restricts how deeply into the broken phase our analysis remains valid, or how small we are permitted to make the scale $\mu$ which we integrate down to. We do not consider this restriction problematic because we are mostly interested in the symmetric phase, and when there is a large condensate we will need to use other tools to determine the rate anyway. We have already considered the broken phase problem in [18].

Under this assumption, the behavior of gauge bosons with $k \gg \mu$ is not significantly changed by a Higgs condensate. It is also not significantly changed by the background of IR Higgs fluctuations, as can be verified by a loopwise analysis like the one in the last section; and the effects of hard Higgs excitations are already included by the HTL effective action. Therefore the integration over these degrees of freedom proceeds as it does in the case without Higgs fields. The integration over gauge and $W$ field degrees of freedom is very nontrivial and has been treated at length by Bödeker [7,8] and by Arnold, Son, and Yaffe [9, 12]. They show that a collision term is induced for the $W$ fields, together with noise 
required by the fluctuation dissipation theorem. The integration over the $g T$ scale Higgs fields is much simpler. The Higgs field equation of motion, Eq. (3.7), is the same below the $g T$ scale as it is up to the $T$ scale. Provided that we keep $\mu \gg m_{H}(T)$, the Higgs field still undergoes free relativistic propagation in the soft gauge and Higgs field background up to corrections $O\left(g^{2} T / \mu\right)$. Such propagation is precisely what generated the hard thermal loops. Standard power counting shows that contributions from the Higgs fields with momentum $k>\mu$ to more IR degrees of freedom are suppressed by $g^{2} T / \mu$ except for UV divergent contributions. But, as shown in [26], the structure of UV divergences in the classical field theory coincides exactly with the hard thermal loops. Hence the $k>\mu$ Higgs degrees of freedom induce HTL's and parametrically suppressed additional effects. The HTL effects from the $g T$ Higgs fields are smaller by a factor of $g$ than those from $T$ physics, so we can neglect them next to the HTL effects already being included.

It is worth remarking why physically no collision integral is induced when we integrate out scalars with momentum $k \sim g T$. The total scattering rate for hard modes by exchange of a soft gauge particle is $\sim g^{2} T \log (1 / g)$, with the logarithm arising from the momentum region $g T$ to $g^{2} T$. This large collision rate originates from the IR singular $s^{2} / t^{2}$ matrix elements in gauge boson exchange. When we integrate out $g T$ bosons we must explicitly include their contribution to scatterings via a collision integral. On the other hand, no scattering process between hard modes which is mediated by single scalar exchange has a scattering rate greater than $\sim g^{4} T \log \left(T / m_{H}\right)$. I Such rare scatterings are not important at leading order because we are eventually interested in physics at the $1 / g^{2} T$ length scale.

On length scales more infrared than $1 / g T$, Eqs. (3.6) and (3.8) describe overdamped evolution for the gauge fields. Further, the $W$ field equation of motion is linear in its slowly varying source $\propto v_{i} E_{i}$. Therefore it is permissible at leading order in $\mu / g T$ to drop both the $D_{t} E$ term in Eq. (3.6) and the $D_{t} W$ term in Eq. (3.8) [5]7], leading to an effective theory for the $\mu \ll g T$ scale physics, 口

$$
\begin{aligned}
m_{\mathrm{D}} \int \frac{d \Omega}{4 \pi} v_{i} W^{a}(x, v) & =-g^{2} \frac{\partial H_{A}}{\partial A_{i}^{a}(x)}-g^{2} \frac{\partial H_{\Phi}}{\partial A_{i}^{a}(x)}, \\
D_{t} \Pi(x) & =-\frac{\partial H_{\Phi}}{\partial \Phi^{\dagger}(x)}, \\
v_{i}\left(D_{i} W\right)^{a}(x, v) & =m_{\mathrm{D}} v_{i} E_{i}^{a}(x)-\int \frac{d \Omega_{v^{\prime}}}{4 \pi} C_{v v^{\prime}} W^{a}\left(x, v^{\prime}\right)+\zeta^{a}(x, v), \\
m_{D} \int \frac{d \Omega_{v}}{4 \pi} W^{a}(x, v) & =0 .
\end{aligned}
$$

The new features are the collision integral $C_{v v^{\prime}}$, whose form and value is discussed in [7, 9, [12], and the noise $\zeta$. At leading order in $\log (g T / \mu)$ the collision integral is

$$
C_{v v^{\prime}} \simeq \gamma\left(\delta_{S^{2}}\left(v-v^{\prime}\right)-\frac{4}{\pi} \frac{\left(v \cdot v^{\prime}\right)^{2}}{\sqrt{1-\left(v \cdot v^{\prime}\right)^{2}}}\right), \quad \gamma \simeq \frac{N_{c} g^{2} T}{4 \pi} \log \frac{m_{D}}{\mu} .
$$

\footnotetext{
${ }^{6}$ This can be verified by looking at the matrix elements of tree level $2 \rightarrow 2$ scattering processes mediated by a scalar, none of which contain $s^{2} / t^{2}$ type terms. At worst they go as $s^{2} / t u$ and are log IR divergent.

${ }^{7}$ For a discussion of the last equation, see [15].
} 
The noise is Gaussian and white with two-point correlator

$$
\left\langle\zeta^{a}(x, v, t) \zeta^{b}\left(y, v^{\prime}, t^{\prime}\right)\right\rangle=2 g^{2} T C_{v v^{\prime}} \delta^{a b} \delta(x-y) \delta\left(t-t^{\prime}\right)
$$

Because of our nonstandard $W$ field normalization, the normalization of the noise differs from the references. Note that the size of the collision integral is parametrically $C_{v v^{\prime}} \sim$ $g^{2} T \log (g T / \mu)$.

At the $k \sim g^{2} T$ scale this effective theory has two natural time scales. First there is the time scale $\sim 1 / g^{2} T$, on which Eq. (3.12) allows the Higgs fields to evolve. There is also a scale, $\sim 1 / g^{4} T \log (1 / g)$, on which the overdamped gauge and $W$ fields evolve. This reflects the fact that the gauge HTL's include Landau damping and lead to overdamped evolution, while the Higgs HTL's are just a mass correction and do not induce any $g^{2} T^{2}$ size damping, so the Higgs field is not overdamped.

If we view the system on length scales $\sim 1 / g^{2} T$ and on the Higgs time scale $\sim 1 / g^{2} T$, the gauge fields look "frozen" up to parametrically suppressed effects. On these time scales the Higgs field evolution is determined by Eq. (3.12) with the gauge field background frozen. What is the behavior of such a system? The Higgs propagates on an inhomogeneous background connection, interacting with itself via the nonlinear quartic coupling term. There is a widely (though not universally) believed conjecture that the evolution of a scalar field theory with quartic self-coupling in 3 dimensions should be ergodic, in which case it will randomize itself on the $1 / g^{2} T$ time scale (since there is no other available time scale for its evolution). This view is supported, for instance, by the calculation of the damping rate of a scalar at rest, in a flat connection but with a quartic interaction; the damping rate is parametrically $\sim \lambda^{2} T^{2} / m_{H} \sim g^{2} T$, and the damping arises primarily from degrees of freedom with $k \sim m_{H}$ [27]. We expect that the inhomogeneous connection should only make the randomization of the scalar field more efficient. In particular we speculate that the spectrum of the $D^{2}$ operator for a typical 3-D gauge field background exhibits Anderson localization at all frequencies.

On the $1 / g^{4} T$ time scale on which the gauge field evolves, the Higgs field will thoroughly explore its fixed connection thermal ensemble. Therefore, on the time scale on which $A$ evolves, it will see a thermodynamic average of the possible Higgs field configurations. We emphasize that we are relying here on the conjectured ergodicity of 3-D scalar $\phi^{4}$ theory. It is also not clear that our treatment will remain true very near the endpoint of the electroweak phase transition, where the Higgs field correlation length grows to be $\gg 1 / g^{2} T$, because in this regime it may take much longer for the Higgs field to explore its fixed gauge field ensemble. We will exclude that regime from consideration, although it is not clear to us that the effective theory we will derive cannot be used there as well.

When our assumption is valid, we should average the $\Phi$ dependent part of Eq. (3.11) over the Higgs thermal ensemble,

$$
-g^{2} \frac{\partial H_{\Phi}}{\partial A_{i}^{a}} \rightarrow-g^{2}\left\langle\frac{\partial H_{\Phi}}{\partial A_{i}^{a}}\right\rangle=g^{2} T \frac{\partial}{\partial A_{i}^{a}} \log \int \mathcal{D} \Phi \exp \left(-H_{\Phi} / T\right) .
$$

The RHS of Eq. (3.11) can be understood as $g^{2} T$ times a variation of a 3-D effective action describing the thermodynamics of the gauge fields. The sole modification from the inclusion of Higgs fields is that the effective action should include, besides the gauge part, a nonlocal 
piece arising from integrating over the Higgs fields. Hence the effective theory describing infrared gauge bosons is

$$
\begin{aligned}
m_{\mathrm{D}} \int \frac{d \Omega}{4 \pi} v_{i} W^{a}(x, v) & =-g^{2} T \frac{\partial}{\partial A_{i}^{a}(x)} \frac{H_{\mathrm{eff}}(A)}{T}, \\
\frac{H_{\mathrm{eff}}(A)}{T} & =-\log \int \mathcal{D} \Phi \exp \left(-\frac{H_{A}+H_{\Phi}}{T}\right), \\
v_{i}\left(D_{i} W\right)^{a}(x, v) & =m_{\mathrm{D}} v_{i} E_{i}^{a}(E)-\int \frac{d \Omega_{v^{\prime}}}{4 \pi} C_{v v^{\prime}} W^{a}\left(x, v^{\prime}\right)+\zeta^{a}(x, v) .
\end{aligned}
$$

This is identical with the "theory 2" of reference [13,12, except for the Higgs field additions to $H_{\text {eff }}$.

This theory is probably not well suited to numerical study. However, as Bödeker has shown, it has fairly simple behavior when studied at the length scale $1 / g^{2} T$ [7]. In this regime the collision integral dominates over the derivative term for the $W$ field 8 , since $C \sim g^{2} T \log (g T / \mu)$ with $\mu \sim g^{2} T$, while the derivative term is $v \cdot D \sim g^{2} T$; the collision term is therefore bigger by $\sim \log (1 / g)$. Roughly speaking, one may solve for $W$ in terms of $E$ in Eq. (3.20) and plug it into Eq. (3.18), yielding a local expression of form $\sigma E=-\partial H / \partial A$.

At leading log order the Yang-Mills theory argument goes over directly to the case including a Higgs field, since it depends on manipulations of Eq. (3.20) only and this does not include the Higgs field. The next to leading log calculation of $[12$ also still holds, provided there is no Higgs condensate of size $\phi_{0} \gtrsim g T / \log (1 / g)$. We discuss this point at more length in an appendix, where we show how the addition of the Higgs field does not modify the calculation presented in [12]. Intuitively, the reason the Higgs field does not affect $\sigma$ at next to leading $\log$ order is as follows. The conductivity depends on the efficiency of collisions. The collisions are mediated by gauge excitations with momenta $g T \gtrsim k \gtrsim g^{2} T \log (1 / g)$. At the low end of this range the Higgs fields modify the gauge field thermodynamics by $O(1 / \log (1 / g))$, see Section [1] and this part of the range gives a contribution down by $1 / \log (1 / g)$ compared to the complete collision integral. Hence the influence of the Higgs field is suppressed by two powers of log.

Finally, we arrive at the effective theory

$$
\begin{aligned}
\sigma E_{i}^{a}(x) & =-g^{2} T \frac{\partial}{\partial A_{i}^{a}(x)} \frac{H_{\mathrm{eff}}(A)}{T}+\xi_{i}^{a}(x) \\
\left\langle\xi_{i}^{a}(x, t) \xi_{j}^{b}\left(y, t^{\prime}\right)\right\rangle & =2 g^{2} T \sigma \delta_{i j} \delta_{a b} \delta^{3}(x-y) \delta\left(t-t^{\prime}\right), \\
\sigma^{-1} & =\frac{3}{m_{D}^{2}} \gamma, \quad \gamma=\frac{N_{\mathrm{c}} g^{2} T}{4 \pi}\left[\ln \frac{m_{D}}{\gamma}+3.041\right] .
\end{aligned}
$$

Here $H_{\text {eff }}$ is as in Eq. (3.19) and the expression for $\sigma^{-1}$ is from [13] with a particularly nice choice for the renormalization scale $\mu$. The sole modification the inclusion of the Higgs fields has made is in the form of $H_{\text {eff }}$. Again we emphasize that the the derivation has assumed that the Higgs is light and that there is not a large Higgs condensate. If there is a large

\footnotetext{
${ }^{8}$ What follows is a gross oversimplification of the argument, see [8,9].
} 
Higgs condensate $\phi_{0} \gtrsim g T / \log (1 / g)$ then the value of $\sigma$ changes, and if $\phi_{0} \sim T$ then the form of the effective theory changes as well. We will not discuss the latter case here. Also note that our effective theory is only valid for studying gauge field correlators; it cannot tell us much about unequal time Higgs field correlators.

\section{NUMERICS}

Bödeker's effective theory is Eq. (3.21), but with $H_{\text {eff }}$ replaced with $H_{A}$. It makes an excellent starting point for numerical investigation of $\Gamma$ in pure Yang-Mills theory for two reasons:

1. it is local, and

2. it is UV finite.

Neither is true for the effective theory we have derived. This potentially makes its study much more problematic than Bödeker's effective theory.

In practice the second problem is not a substantial one. The theory is not UV finite because $H_{\text {eff }}$ involves the 3 dimensional path integral for a scalar field, which contains linear and logarithmic mass divergences arising from one and two loop graphs. However, the path integral is still super-renormalizable, and the UV infinities are purely local and can be absorbed with a mass counterterm. Their value is known [28,29], and as we will discuss below, we could actually proceed even if they were not.

The real problem with implementing the effective theory is its nonlocality, which comes about because of the path integral in the expression for $H_{\text {eff }}$, see Eq. (3.19). The solution is to think about how we would carry out such a path integral numerically. To evaluate Eq. (3.19) numerically, we would perform the path integral by Monte-Carlo, for instance by a Langevin equation,

$$
\begin{aligned}
\frac{d \Phi}{d \tau_{\phi}}(x) & =-\frac{\partial H_{\Phi}}{\partial \Phi^{\dagger}(x)}+\xi(x), \\
\left\langle\xi\left(x, \tau_{\phi}\right) \xi^{\dagger}\left(x^{\prime}, \tau_{\phi}^{\prime}\right)\right\rangle & =2 T \mathbf{1} \delta\left(x-x^{\prime}\right) \delta\left(\tau_{\phi}-\tau_{\phi}^{\prime}\right) .
\end{aligned}
$$

Here the 1 reminds us to make the noise diagonal in the components of the Higgs field. This Langevin equation must be evolved, and the result averaged, at every time step in the Langevin dynamics of the gauge fields.

This suggests that Eq. (3.21) can be replaced with the $\eta \rightarrow \infty$ limit of the following system of equations:

$$
\begin{aligned}
\sigma E_{i}^{a}(x) & =-g^{2} \frac{\partial}{\partial A_{i}^{a}(x)}\left(H_{A}(A)+H_{\Phi}(A, \Phi)\right)+\xi_{i}^{a}(x, t), \\
\sigma D_{t} \Phi(x) & =-\eta \frac{\partial}{\partial \Phi^{\dagger}(x)} H_{\Phi}(A, \Phi)+\xi_{\Phi}(x, t), \\
\left\langle\xi_{\Phi}(x, t) \xi_{\Phi}^{\dagger}\left(x^{\prime}, t^{\prime}\right)\right\rangle & =2 \eta \sigma T \mathbf{1} \delta\left(x-x^{\prime}\right) \delta\left(t-t^{\prime}\right) .
\end{aligned}
$$

This is Langevin evolution but with a different rate for the evolution of the two fields, $A$ and $\Phi$. In the limit that the Higgs field evolution is made infinitely fast, which is $\eta \rightarrow \infty$, 
the Higgs field evolution will perform the path integral in a much shorter time scale than the gauge fields evolve in, and we recover the desired equations of motion, Eq. (3.21).

Eq. (4.3) at a finite value of $\eta$ does make a good starting point for numerical work. The large $\eta$ limit must then be taken numerically. It is also convenient to rescale time to a Langevin time, $\tau \equiv \sigma t$. Note that the dimensions of $\tau$ are those of a length squared.

Our nonperturbative regularization for Eq. (4.3) will be the lattice. Since all of the numerical tools we use exist in the literature, we will only present the relevant references here rather than give complete details. The lattice discretization is standard, see [30]. Our topological lattice definition of $N_{\mathrm{CS}}$ is the same as in [31]. The relation between the couplings of the lattice and continuum systems has been worked out in [32], and we use the expressions there. These relations match all thermodynamic quantities at $O(a)$, leaving $O\left(a^{2}\right)$ errors, with two exceptions. We do not know the full $O(a)$ match for an additive piece of the $\phi^{2}$ operator insertion or for $m_{H}^{2}(T)$. If we were interested in determining $\Gamma$ at a particular, fixed value of $m_{H}^{2}(T)$ then this could pose a problem. However, what we want is $\Gamma$ when $m_{H}^{2}(T)$ is a fixed distance from the value which gives phase equilibrium, $m_{\text {eq }}^{2}$. To determine this we do not need the absolute normalization of $m_{H}^{2}(T)$ to $O(a)$, but we will need to find $m_{\text {eq }}^{2}$ numerically.

Now we briefly discuss algorithm. To determine $m_{\mathrm{eq}}^{2}$ we use the same algorithm and multicanonical techniques as [30]. To perform Eq. (4.3), we may use whatever Higgs update we choose, provided that it is stochastic and that we take the $\eta \rightarrow \infty$ limit. We choose a mixture of heat bath Higgs updates and the $\mathrm{x}-\mathrm{y}$ over-relaxation algorithm of [30]. For the gauge field update we should use Langevin dynamics or any other strictly dissipative dynamics. We choose heat bath. For either algorithm, the effective infrared dynamics should be of the Langevin form, and the difference between the algorithms will be a radiative rescaling of the (Langevin) time scale and high dimension corrections, which first appear at

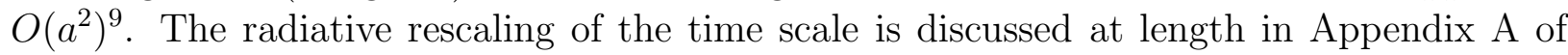
14]. That paper presents an analytic calculation of the lattice to continuum match for the Langevin algorithm, and the Langevin to heat-bath rescaling is found numerically. Within error it is equal to the coefficient $Z_{g}^{-1}$ which appears in the $O(a)$ thermodynamical match detailed in [32]. We will assume that this numerically determined relation holds analytically.

\section{RESULTS}

With a numerical implementation of the effective theory in hand we will address two questions. First, how large is $\Gamma$ in the symmetric phase, at $m_{\mathrm{eq}}^{2}$, for parameters where the phase transition is strong enough to preserve baryon number after its completion? Second, how does $\Gamma$ vary as we go through the analytic crossover present for large vacuum Higgs masses?

To address the first question we consider Yang-Mills Higgs theory at $\lambda / g^{2}=0.036$, which is roughly the value at which the phase transition is barely strong enough to preserve baryon

\footnotetext{
${ }^{9}$ The question of what modifications may occur in the effective IR behavior has been addressed at some length by Arnold and Yaffe, see particularly Appendix A of [15]
} 

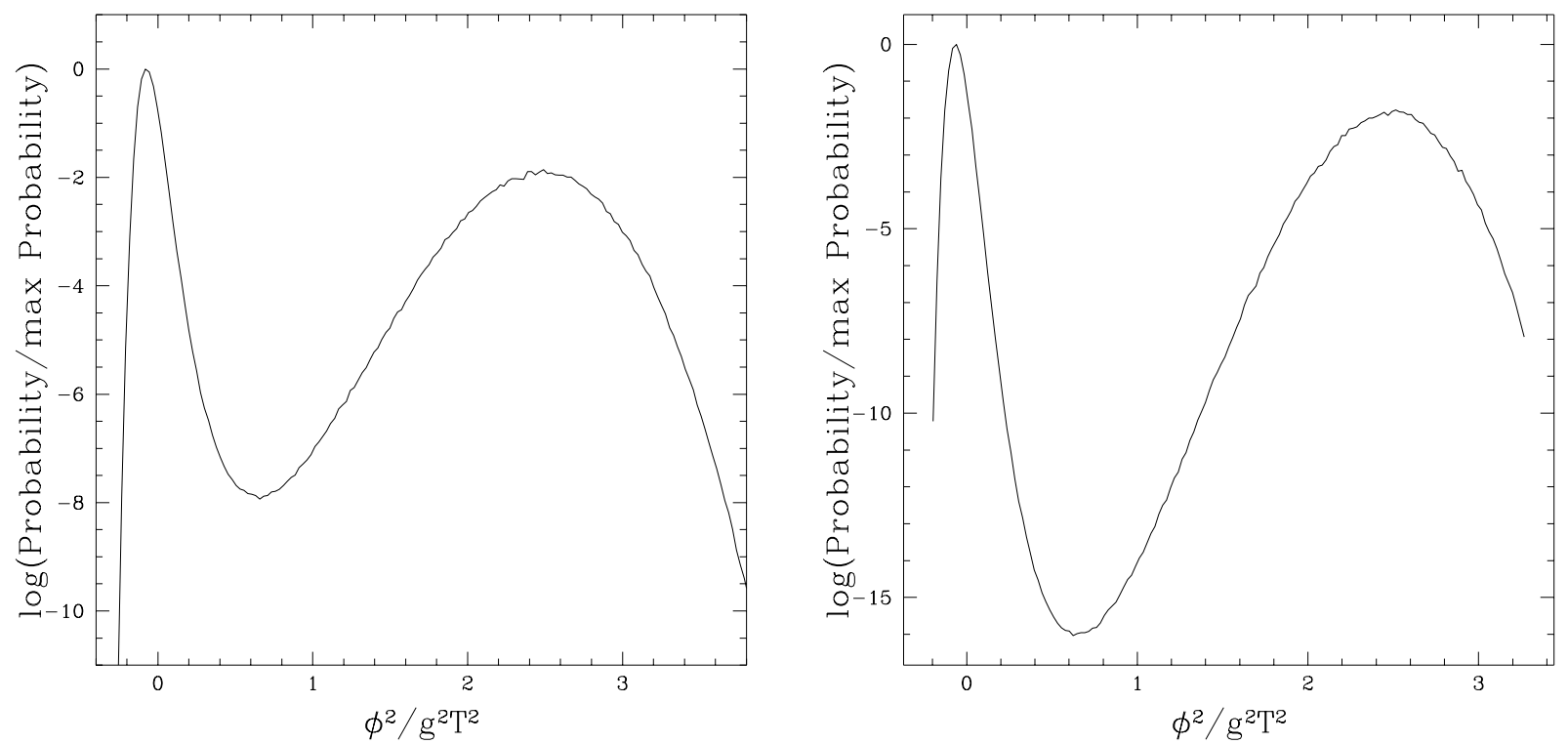

FIG. 2. Log of probability distribution for $\phi^{2} \equiv 2 \Phi^{\dagger} \Phi\left(\overline{\mathrm{MS}}\right.$ renormalization point $\left.\bar{\mu}=g^{2} T\right)$ for cubic boxes of size $24^{3}$ (left) and $32^{3}$ (right), at lattice spacing $4 / 9 g^{2} T(\beta=9)$. Inverting the $y$ axis roughly gives the free energy dependence on $\phi^{2}$. The left peak is the symmetric phase and the right peak is the broken phase; the dip between them is a free energy barrier separating the phases, which grows as the volume is increased (it is not free energy per volume). The asymmetric shape is typical for this order parameter.

number cosmologically after its completion. We determine $m_{\mathrm{eq}}^{2}$ at three lattice spacings, $a=4 / 7 g^{2} T, a=4 / 9 g^{2} T$, and $a=4 / 12 g^{2} T$ (which in the notation which is sometimes customary [33] are $\beta=7,9$, and 12), by multicanonical Monte-Carlo. The probability distributions for $\phi^{2}$ at $m_{\mathrm{eq}}^{2}$ for two lattice volumes and $a=4 / 9 g^{2} T$ are shown in Fig. 2 .

First we consider the $\eta \rightarrow \infty$ limit at fixed lattice spacing $a=4 / 9 g^{2} T$ in a cubic volume $L^{3}$ with periodic boundary conditions, for $L=14.2 / g^{2} T$ (32 sites on a side). This volume is abundantly large enough to see the large volume value of $\Gamma$ [31], and is large enough that strong metastability prevents tunneling to the broken phase at $m_{\text {eq }}^{2}$, where we work. Table Q 0 presents $\kappa^{\prime}$, defined in Eq. (1.3), for various values of $\eta$, lattice spacing, and $m_{H}^{2}$. The $\eta$ dependence is weak and statistically compatible with zero.

\begin{tabular}{|c|c|c|c|c|}
\hline$a \times g^{2} T$ & $\eta$ & $m_{H}^{2}-m_{\text {eq }}^{2}$ & volume $\times$ Langevin time & $\kappa^{\prime}$ \\
\hline $4 / 9$ & 2.5 & 0 & $32^{3} \times 56400$ & $8.45 \pm .24$ \\
\hline $4 / 9$ & 5 & 0 & $32^{3} \times 56400$ & $8.20 \pm .24$ \\
\hline $4 / 9$ & 10 & 0 & $32^{3} \times 56400$ & $8.08 \pm .23$ \\
\hline $4 / 9$ & 5 & .0093 & $32^{3} \times 34300$ & $8.63 \pm .32$ \\
\hline $4 / 9$ & 5 & -.0093 & $32^{3} \times 30900$ & $8.50 \pm .33$ \\
\hline $4 / 7$ & 10 & 0 & $24^{3} \times 57400$ & $8.13 \pm .37$ \\
\hline $4 / 12$ & 5 & 0 & $40^{3} \times 98600$ & $7.83 \pm .27$ \\
\hline $4 / 9$ & Yang & Mills & $24^{3} \times 489400$ & $9.90 \pm .13$ \\
\hline $4 / 10$ & Yang & Mills & $40^{3} \times 84900$ & $10.00 \pm .23$ \\
\hline
\end{tabular}

\footnotetext{
${ }^{10}$ The value we use is slightly smaller than that found in [18] because here we do not include the U(1) field.
} 

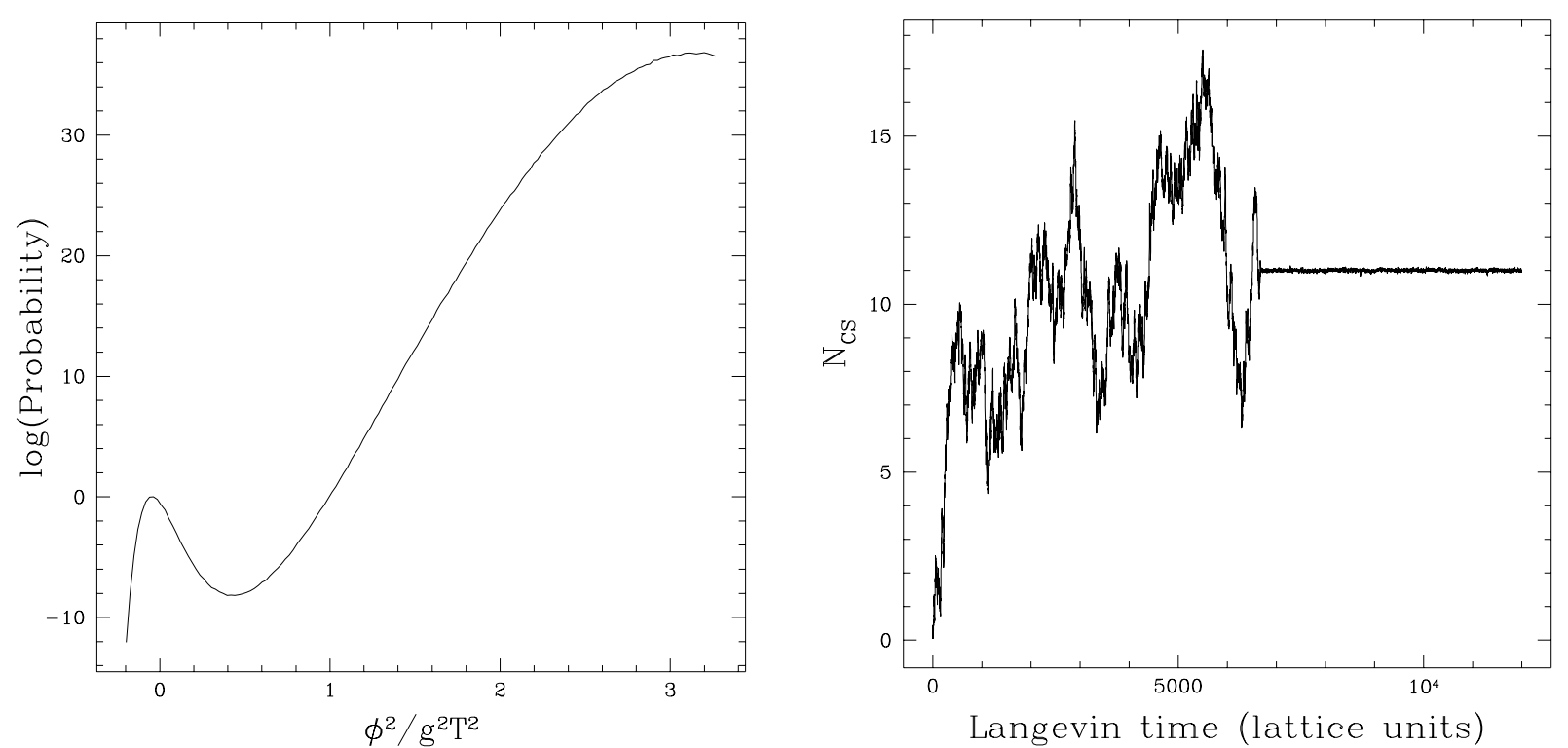

FIG. 3. Left: probability distribution on supercooling, $m_{H}^{2}=m_{\mathrm{eq}}^{2}-.0093 g^{4} T^{2}$. Right: Langevin time history of $N_{\mathrm{CS}}$ starting in the symmetric phase, under such supercooling. After a tunneling event to the broken phase, $N_{\mathrm{CS}}$ stopped diffusing.

TABLE I. Chern-Simons number diffusion $\Gamma$ in the symmetric phase near the equilibrium temperature with $\lambda / g^{2}=.036$, for different lattice spacings, values of $\eta$, and Higgs masses. The 4 -volume is expressed in lattice units; to convert to physical units multiply lengths by $a$ and Langevin time by $\sigma a^{2}$. Except for the pure Yang-Mills data, all values of $\kappa^{\prime}$ are statistically compatible; no lattice or $\eta$ dependence is statistically significant.

Table 1 also shows that, as expected, there is almost no lattice spacing dependence in $\Gamma$. The $O(a)$ match for the thermodynamic quantities and time scales is essential here, see [14]. Finally, since in the usual electroweak baryogenesis scenario the symmetric phase undergoes supercooling, we should study $\Gamma$ in the supercooled symmetric phase. Table प shows that the maximum supercooling compatible with strong metastability is still not enough to significantly change $\Gamma$. We cannot increase the supercooling beyond what was used because the lattice system will nucleate to the broken phase. Indeed, one run used for the table ended with a nucleation to the broken phase, as is seen clearly from the time history for $N_{\mathrm{CS}}$ in that run, shown in Fig. 3. Naturally, the broken phase part of the evolution was not used in the analysis. Combining all the figures in the table, since all are statistically compatible and no trend (lattice spacing, supercooling, or $\eta$ ) is statistically significant, we get

$$
\Gamma_{\text {symm }} \simeq[8.24 \pm 0.10]\left(\frac{g^{2} T^{2}}{m_{D}^{2}}\right)\left(\log \frac{m_{D}}{g^{2} T}+C\right) \alpha^{5} T^{4}
$$

for $\lambda / g^{2}=0.036$. We have computed the pure Yang-Mills theory value at the same lattice spacing with comparable precision to facilitate comparison; the symmetric phase value is $0.832 \pm .015$ of the Yang-Mills theory value. (The Yang-Mills theory value found here is smaller than that quoted in [14], where we found $\kappa^{\prime}=10.8 \pm 0.7$. This is a statistical fluctuation in the data there. The table includes a higher statistics redetermination of $\kappa^{\prime}$ at the same parameters used in that paper, with a result in agreement with the $\beta=9$ value found here, and statistically compatible at $1 \sigma$ with the result determined in [14].) 

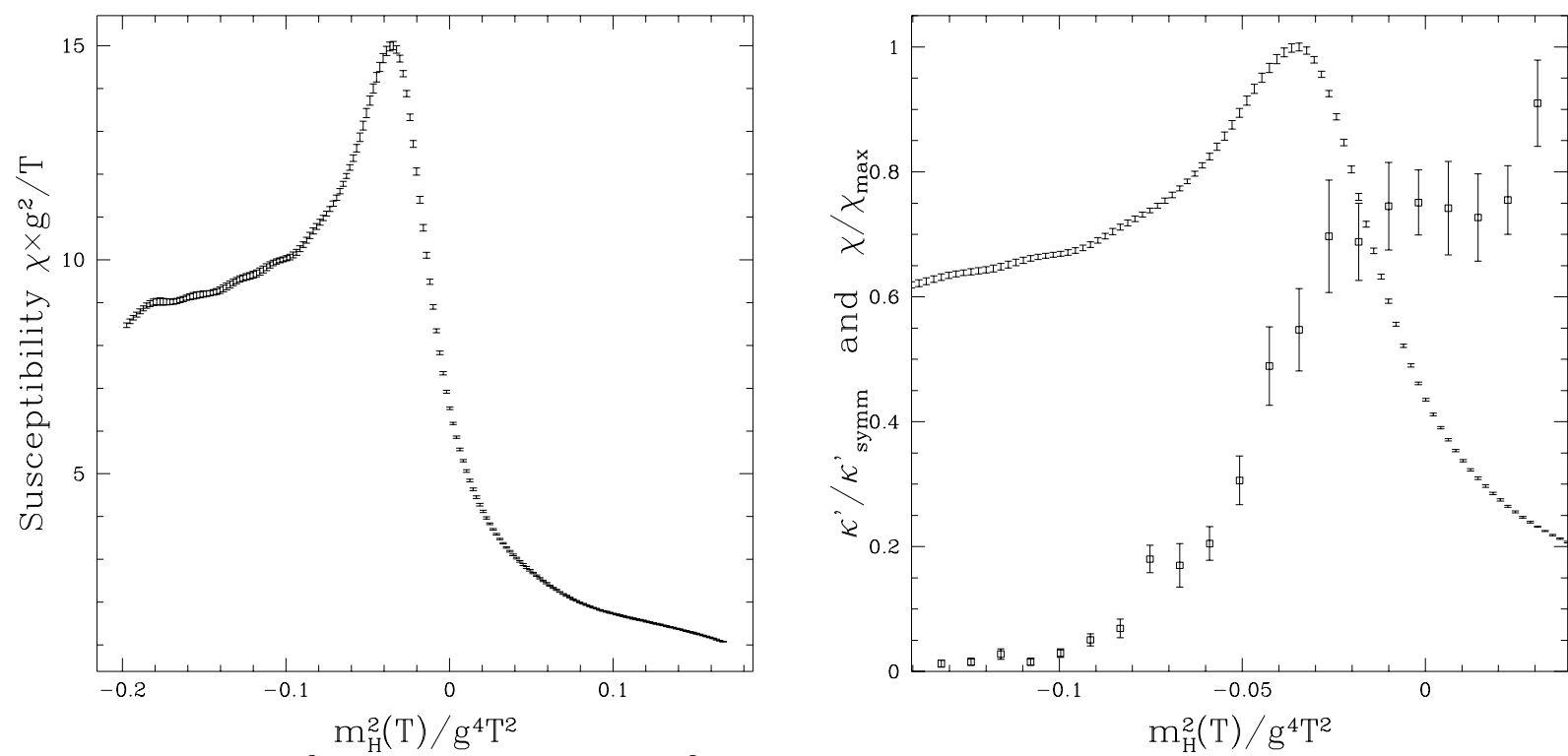

FIG. 4. Left: $\phi^{2}$ susceptibility; Right: $\phi^{2}$ susceptibility (peak with small errors) and sphaleron rate (larger errors, square plotting symbols) scaled to their maxima, when there is a smooth crossover. $m_{H}^{2}(T)$ plotted is the $3 \mathrm{D}$ theory value, $\overline{\mathrm{MS}}$ renormalized with $\bar{\mu}=g^{2} T$.

Of course the above is academic because $\lambda / g^{2}=0.036$ converts to a vacuum Higgs mass $m_{H}=44 \mathrm{GeV}$ at tree level. Including the large radiative top Yukawa corrections, this $\lambda / g^{2}$ does not correspond to any physical Higgs mass [16]. In the standard model, all experimentally allowed Higgs masses fail to provide an electroweak phase transition at all. Rather, there is an analytic crossover [34]. Although baryogenesis is probably impossible in that setting, it would still be interesting to see how $\Gamma$ varies as we go through the crossover. Does it turn on suddenly or gradually, and does its turn-on point coincide with the peak in the susceptibility for $\phi^{2}$ ? We answer this question in Fig. 囷, which shows how the $\phi^{2}$ susceptibility

$$
\chi_{\phi^{2}} \equiv \frac{1}{V}\left[\left\langle\left(\int \phi^{2}\right)^{2}\right\rangle-\left\langle\int \phi^{2}\right\rangle^{2}\right],
$$

and the sphaleron rate $\Gamma$ vary with $m_{H}^{2}(T)$. The data are for $\lambda / g^{2}=5 / 16$, corresponding at tree level to a physical vacuum $m_{H}=130 \mathrm{GeV}$, and were taken for a $32^{3}$ box with lattice spacing $a=1 / 2 g^{2} T$. The sphaleron rate data were all taken using $\eta=20$. We see that the switch-on of $\Gamma$, though smooth, is fairly rapid and occurs at slightly lower $m_{H}^{2}(T)$ than the peak susceptibility; that is, it is when conditions are a little more "broken phase-like" than when the susceptibility peaks. The sphaleron rate $\Gamma$ proves a rather good order parameter to distinguish Higgs-like and symmetric-like phases. Although, like any order parameter must, it shows smooth behavior, the range where it is far from both its broken phase value $\kappa^{\prime} \simeq 0$ and its symmetric phase value $\kappa^{\prime}>5$ is quite narrow, roughly as narrow as the peak in the $\phi^{2}$ susceptibility. However, we do not view the sphaleron rate as competitive with the susceptibility as a probe of where the crossover occurs. The main reason is that it is possible to perform a multicanonical reweighting which allows the susceptibility to be scanned in a wide range of $m_{H}^{2}$ (the data in the figure come from a single numerical run), and the statistics for the susceptibility improve more quickly. For instance, the $N_{\mathrm{CS}}$ diffusion data in Fig. 目 
took more CPU time than the susceptibility data, but are substantially "dirtier". (The data sets are plotted with their error bars. The $N_{\mathrm{CS}}$ diffusion errors are each independent, but there is very large cross-correlation in errors of neighboring points for the susceptibility because they were all computed from one data set.)

\section{CONCLUSION}

Assuming that classical 3-D scalar $\phi^{4}$ theory is ergodic, the addition of a light Higgs degree of freedom replaces Bödeker's effective theory for the evolution of infrared gauge fields with a slightly more complicated equation, Eq. (3.21). The sole change is in the thermodynamic potential for the gauge fields. Including the Higgs field makes this thermodynamic potential nonlocal. However, the effective theory is still a useful starting point for numerical work because we can use the limit of a sequence of local effective theories, namely, Langevin (or heat bath) evolution for the gauge fields and for the Higgs fields, but with much faster Langevin evolution for the Higgs degrees of freedom.

When the electroweak phase transition is strong, the sphaleron rate in the symmetric phase is reduced by around $20 \%$ from its Yang-Mills theory value, roughly in accord with an estimate based on the thermodynamics. (The thermodynamic estimate for small $\lambda / g^{2}$ is $(\delta \Gamma / \Gamma) \simeq 1.2 \sqrt{\lambda / g^{2}}$, see Eq. (2.10). $)$ When there is no electroweak phase transition, but an analytic crossover, the sphaleron rate changes rather quickly from its symmetric phase value to nearly zero, roughly at the same value of $m_{H}^{2}(T)$ where the $\phi^{2}$ susceptibility peaks. The crossover region is of about the same width as the peak in the $\phi^{2}$ susceptibility and is displaced to slightly lower $m_{H}^{2}(T)$.

In this paper we have only studied the standard model, either for parameters which are ruled out experimentally or for which the electroweak phase transition cannot provide for baryogenesis. However it is fairly simple to see how to extend the work to more viable models like the MSSM (minimal supersymmetric standard model). In that case, the SU(2) and SU(3) gauge fields would each evolve under Langevin dynamics, with a thermodynamic potential arising from integrating over the Higgs and scalar top fields. However, from our results with a strong phase transition, it should be clear that, in all cases where the phase transition is strongly first order, $\Gamma$ in the symmetric phase will be very close to its Yang-Mills theory value. This is because we have found that the suppression of $\Gamma$ corresponds well to what we expect thermodynamically; and when the electroweak phase transition is strong in the MSSM, the symmetric phase Higgs mass is larger than in the standard model case. Therefore the SU(2) thermodynamics in the symmetric phase is closer to Yang-Mills theory in the MSSM than in the standard model, see Eq. (2.6). Hence it is almost certainly true that, in the MSSM and when the phase transition is strong, the symmetric phase sphaleron rate is lower than but within $20 \%$ of the pure Yang-Mills theory value. In practice this means we can continue to quote the Yang-Mills theory result for all symmetric phase cases of physical interest, with modest error. 


\section{ACKNOWLEDGMENTS}

I am grateful to Dam Son for a useful discussion in which he conjectured that the Higgs fields would enter only through $H_{\text {eff }}$. I also acknowledge useful conversations with Larry Yaffe and Dietrich Bödeker. This work was partially supported by the DOE under contract DE-FGO3-96-ER40956.

\section{APPENDIX A: HIGGS FIELDS AND HARD THERMAL LOOPS}

In this appendix we show that the only hard thermal loop needed for the bosonic effective theory, which has soft external scalar field lines, is the scalar mass correction.

We need only consider HTL's with bosonic external lines, since the appropriate IR effective theory we seek is bosonic. Hence we need in general to consider all diagrams with $\left(n_{s}>0\right.$, even) scalar and $n_{g}$ gauge boson external lines. We will use repeatedly the power counting rules derived in [20], which we repeat here for the reader's convenience. A hard thermal loop is always an $O\left(T^{2}\right)$ contribution from the $K \sim T$ momentum region of a one loop integral with soft $(g T)$ external lines. To determine the largest power of $T$ possible from a diagram, follow these rules:

1. the loop integration contributes $T^{3}$;

2. the first propagator times the Matsubara sum contributes $1 / T$;

3. every additional propagator contributes $1 /(P T)$;

4. powers of $K^{\mu}$ in the numerator (from 3 point gauge vertices or fermionic propagators) each contribute $T$;

5. when there are two or more propagators, and all propagators are either bosonic or fermionic, there is an extra $P / T$ suppression.

If the result has a weaker power of $T$ than $T^{2}$ the diagram does not contribute an HTL; if it is $T^{2}$ the diagram will unless there is some cancellation.

Since we are only concerned with HTL's with bosonic external legs, then either all the propagators in the loop will be bosons or all will be fermions. Any diagram with more than 1 propagator will get a $P / T$ from rule 5 . and this will exclude diagrams with 4 point vertices ( 4 gluon, 2 gluon and 2 scalar, or 4 scalar) except for tadpoles, just as is the case in Yang-Mills theory. This leaves all diagrams of the general form of diagrams (c) and (d) in Figure 0 .

The scalar self-energy diagrams are considered in [35] (fermion loop) and [36] (boson loops). The result is that the self-energy is a momentum independent mass correction. In particular it is useful to reproduce the argument for the gauge HTL from diagram (a) of Figure 5. In Feynman gauge this diagram contributes (following the notation of that paper, where capital letters are 4-vectors and lower case are spatial or temporal (0 subscript) components) 


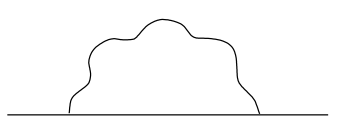

(a)

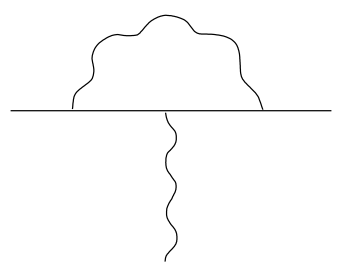

(b)

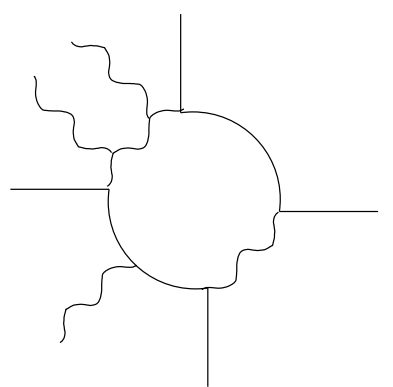

(c)

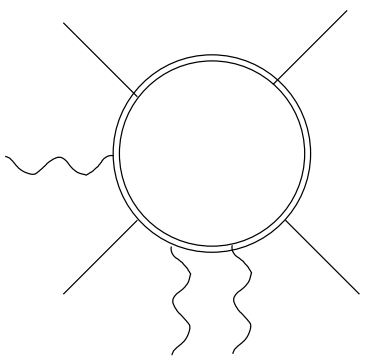

(d)

FIG. 5. Diagrams considered in Appendix A. Solid lines are scalars, wiggly lines are gauge bosons, double lines are fermions. Diagrams (a) and (b) are a self-energy and a vertex correction considered in the text. Diagrams (c) and (d) are generic diagrams with external gauge and scalar lines and either a boson (alternating Higgs and gauge, diagram (c)) or a fermion (diagram (d)) in the loop. As discussed, (b), and all diagrams of type (c) and (d), do not give rise to hard thermal loops.

$$
\begin{aligned}
& -\frac{N^{2}-1}{2 N} g^{2} T \sum_{k_{0}} \int \frac{d^{3} k}{(2 \pi)^{3}} \frac{(2 P+K)^{\mu}(2 P+K)^{\nu} g_{\mu \nu}}{K^{2}(K+P)^{2}} \\
= & -\frac{N^{2}-1}{2 N} g^{2} T T \sum_{k_{0}} \int \frac{d^{3} k}{(2 \pi)^{3}}\left[\frac{2(K+P)^{2}-K^{2}+2 P^{2}}{K^{2}(K+P)^{2}}\right] .
\end{aligned}
$$

For the first term in the square brackets, the $(K+P)^{2}$ in the numerator cancels a propagator in the denominator, leaving one propagator and no powers of $K$ in the numerator. This gives an HTL effect only because there is a single propagator left, so 5. above is not invoked. The result is a momentum independent tadpole. The second term is similar. The last term has two propagators and no power of $K$ in the numerator; it is $O\left(T^{0}\right)$ and does not give an HTL.

Similarly, diagram (b) gives, in Feynman gauge and labeling the incoming scalar momentum $P_{1}$, the incoming gauge momentum $P_{2}$, and the outgoing scalar momentum $P_{3}=P_{1}+P_{2}$,

$$
\propto g^{3} T \sum_{k_{0}} \int \frac{d^{3} k}{(2 \pi)^{3}} \frac{\left(K+2 P_{1}\right) \cdot\left(K+2 P_{3}\right)\left(2 K+P_{1}+P_{3}\right)^{\mu}}{K^{2}\left(K+P_{1}\right)^{2}\left(K+P_{3}\right)^{2}} .
$$

It is convenient to rewrite

$$
\left(K+2 P_{1}\right) \cdot\left(K+2 P_{3}\right)=\left(K+P_{1}\right)^{2}+\left(K+P_{3}\right)^{2}-K^{2}+\left(4 P_{1} \cdot P_{3}-P_{1}^{2}-P_{3}^{2}\right) .
$$

Each $K^{2}$ type term will cancel a propagator, leaving two propagators and one $K^{\mu}$ in the numerator. By the power counting rules this contributes at most $T^{3}(1 / T)(1 / P T) T(P / T) \propto$ $T^{1}$ and does not give a hard thermal loop. The $P^{2}$ term is $O\left(T^{0}\right)$, so it does not either.

This example illustrates why diagrams of form (c) and (d), with $\left(n_{s}>0\right.$, even) scalars and $n_{g}$ gauge bosons, $\left(n_{s}+n_{g}\right)>2$, do not give hard thermal loops. Such a diagram has $\left(n_{s}+n_{g}\right)>2$ propagators. It also has $n_{s}+n_{g}$ factors of $K^{\mu}$ in the numerator, either all from gauge vertices (bosonic loops) or all from fermionic propagators (fermionic loops). However, there are only $n_{g}$ external Lorentz indices. In Feynman gauge, the diagram will be a sum of terms, with at least $n_{s}$ of the $K^{\mu}$ contracted against each other in each term. Hence, each term from each diagram contains in its numerator, in Feynman gauge, a term 
of form $\left(K+P_{i}\right) \cdot\left(K+P_{j}\right)$, with $P_{i}, P_{j}$ some linear combinations of external momenta and $K$ the loop momentum. This can always be written as $\sum(\operatorname{sign})\left(K+P_{l}\right)^{2}+O\left(P^{2}\right)$, with each $\left(K+P_{l}\right)$ a momentum on some propagator. Consider one of the $\left(K+P_{l}\right)^{2}$ terms. Cancelling the $\left(K+P_{l}\right)^{2}$ against the appropriate propagator leaves $\left(n_{s}+n_{g}-2\right) K^{\mu}$ factors in the numerator but $\left(n_{s}+n_{g}-1\right)>1$ propagators. The power counting then gives at most $T^{3}(1 / T)(1 / P T)^{n_{s}+n_{g}-2} T^{n_{s}+n_{g}-2}(P / T) \propto T^{1}$, too small to contribute an HTL, even without further cancellations. Meanwhile, the $O\left(P^{2}\right)$ term has $\left(n_{s}+n_{g}\right)$ propagators but $\left(n_{s}+n_{g}-2\right)$ $K^{\mu}$ factors; it is at most $P^{2} T^{3}(1 / T)(1 / P T)^{n_{s}+n_{g}-1} T^{n_{s}+n_{g}-2}(P / T) \propto T^{0}$ and also gives no HTL. Though we worked in Feynman gauge, we expect the hard thermal loops to be gauge invariant; if they vanish except a momentum independent scalar mass in Feynman gauge, they should in any gauge.

Hence the only bosonic HTL with scalar external lines is the gauge field mass insertion. Note that similar reasoning to what we give above also rules out HTL's with both scalar and fermionic external lines; but this is unimportant for the purposes of this paper.

\section{APPENDIX B: HIGGS FIELDS AND NEXT TO LEADING LOG CONDUCTIVITY}

In this appendix we briefly explain how the calculation presented in [12 is modified by the presence of Higgs fields, and why the modification does not change the conductivity of the final effective theory at next to leading log order. The purpose of the appendix is to outline the argument, at times we will be sloppy with notations and with terms which are not relevant and have been discussed at much more length in [15, 13, 12.

The relevant part of the calculation in [12 is the match between their "theory 2" and "theory 3", only now "theory 2" is given by Eqs. (3.18-3.20), which for convenience we repeat here:

$$
\begin{aligned}
m_{\mathrm{D}} \int \frac{d \Omega}{4 \pi} v_{i} W^{a}(x, v) & =-g^{2} T \frac{\partial}{\partial A_{i}^{a}(x)} \frac{H_{\mathrm{eff}}(A)}{T} \\
\frac{H_{\mathrm{eff}}(A)}{T} & =-\log \int \mathcal{D} \Phi \exp \left(-\frac{H_{A}+H_{\Phi}}{T}\right) \\
v_{i}\left(D_{i} W\right)^{a}(x, v) & =m_{\mathrm{D}} v_{i} E_{i}^{a}(E)-\int \frac{d \Omega_{v^{\prime}}}{4 \pi} C_{v v^{\prime}} W^{a}\left(x, v^{\prime}\right)+\zeta^{a}(x, v) .
\end{aligned}
$$

It is possible, at least formally, to invert Eq. (B3) to solve for $W$ in terms of $E$. Eq. (B1) then becomes

$$
\left(\sigma_{i j}(D) E_{j}\right)(x)=-g^{2} T \frac{\partial}{\partial A_{i}(x)} \frac{H_{\mathrm{eff}}(A)}{T}+\zeta_{i}^{\prime}
$$

where $\sigma(D)$ is the nonlocal operator resulting from inverting Eq. (B3); it is discussed in [12]. $\zeta^{\prime}$ is a noise with correlator $\left\langle\zeta^{\prime} \zeta^{\prime}\right\rangle=2 T \sigma(D)$. (Starting here we will aggressively suppress indices where we feel the meaning is clear.)

This Langevin equation only has a path integral representation if we are willing to accept $\left(\delta H_{\text {eff }} / \delta A\right)^{2}$ inside the action of a path integral. It is not clear how to form a perturbation 
theory for such a path integral. However, if we rewrite $H_{\text {eff }}$ as we did in Section IV], then it will become possible. The effective theory of interest is the $\eta \rightarrow \infty$ limit of

$$
\begin{aligned}
\sigma_{i j}(D) E_{j} & =-g^{2} T\left(\frac{\partial H_{A}}{\partial A_{i}}+\frac{\partial H_{\Phi}}{\partial A_{i}}\right), \\
D_{t} \Phi & =-\eta \frac{\partial H_{\Phi}}{\partial \Phi^{\dagger}}+\xi \\
\left\langle\xi(x, t) \xi^{\dagger}\left(x^{\prime}, t^{\prime}\right)\right\rangle & =2 \eta T \mathbf{1} \delta\left(x-x^{\prime}\right) \delta\left(t-t^{\prime}\right) .
\end{aligned}
$$

To write a path integral expression for this, we follow the standard trick of writing the Langevin equation and the average over the noise distribution as a path integral,

$$
\begin{aligned}
\int \mathcal{D} \zeta^{\prime} \mathcal{D} \xi \mathcal{D} A \mathcal{D} \Phi \exp \left(-\int \zeta^{\prime} \frac{1}{4 g^{2} \sigma(D) T} \zeta^{\prime}\right) \exp \left(-\int \xi \frac{1}{4 \eta T} \xi\right) \\
\times \delta\left(\zeta^{\prime}+\sigma(D) E+g^{2} T\left[\delta\left(H_{A}+H_{\Phi}\right) / \delta A\right]\right) \delta\left(\xi+D_{t} \Phi+\eta\left[\delta H_{\Phi} / \delta \Phi^{\dagger}\right]\right),
\end{aligned}
$$

times Jacobians for each field, which are not important, see 15,12]. Here the path integral performs the average over the noise, and the delta functions enforce the Langevin equations. Enforcing the delta function does the integrals over each noise, giving a path integral

$$
\begin{aligned}
& \int \mathcal{D} A \mathcal{D} \Phi \exp (-L), \\
L= & \left(\sigma(D) E+g^{2} T\left[\frac{\delta\left(H_{A}+H_{\Phi}\right)}{\delta A}\right]\right) \frac{1}{4 g^{2} \sigma(D) T}\left(\sigma(D) E+g^{2} T\left[\frac{\delta\left(H_{A}+H_{\Phi}\right)}{\delta A}\right]\right) \\
& +\left(D_{t} \Phi+\eta\left[\frac{\delta H_{\Phi}}{\delta \Phi^{\dagger}}\right]\right)^{\dagger} \frac{1}{4 \eta T}\left(D_{t} \Phi+\eta\left[\frac{\delta H_{\Phi}}{\delta \Phi^{\dagger}}\right]\right) .
\end{aligned}
$$

Here we have not written the contributions from the Jacobians or from an extra regulation dependent term arising because of the nonlocality of $\sigma$, which ref. [12] calls $L_{1}[A]$. These terms, and the reasons they can be dropped, are discussed at some length in 15:12].

Arnold and Yaffe have shown that $\sigma$ in Eq. (3.21) can be determined at next to leading $\log$ order by computing the $\omega=0, k \rightarrow 0$ limit of the one loop, Coulomb gauge $A_{0} A_{0}$ self-energy for the theory with $\sigma(D)$, and finding what constant value of $\sigma$ is needed to get the same one loop result in the final effective theory. To account for Higgs contributions in this calculation, we must find all one loop Higgs field self-energy corrections to the $A_{0}$ field, and must determine whether their contributions survive in the $\eta \rightarrow \infty$ limit.

First we have to find what vertices couple $\Phi$ to $A_{0}$. The only terms in the Lagrangian which contain $A_{0}$ at all are those which contain $E$ or $D_{t}$. They are

$$
\frac{1}{4} E \sigma(D) E+\frac{1}{4 \eta}\left(D_{t} \Phi\right)^{\dagger}\left(D_{t} \Phi\right)
$$

plus terms odd in $D_{t}$, which are of form

$$
\frac{1}{2} E_{i} \frac{\delta H_{A}}{\delta A_{i}}, \quad \frac{1}{4}\left(2 E_{i} \frac{\delta H_{\Phi}}{\delta A_{i}}+\left(D_{t} \Phi\right)^{\dagger} \frac{\delta H_{\Phi}}{\delta \Phi^{\dagger}}+c c\right) .
$$


The first term here is proportional to $\partial_{t} H_{A}$. The second is proportional to $\partial_{t} H_{\Phi}$. Both are total spacetime derivatives, which can be converted to boundary terms at asymptotically early and late times and therefore neglected. Hence we only need to consider vertices which arise from the terms in Eq. (B10). These allow two new diagrams not considered in [12, which are the same as (a) and (b) in Figure 11 if the wavy lines are now taken to be $A_{0}$ propagators.

To show that these diagrams vanish in the small $\eta$ limit, we need only count powers of $\eta$ in the vertices and propagators. The $A_{0} \Phi^{2}$ vertex carries a factor of $\omega / \eta$ and the $A_{0}^{2} \Phi^{2}$ vertex carries a factor of $1 / \eta$. The $\Phi$ propagator is

$$
\langle\Phi \Phi\rangle=\frac{1}{\omega^{2} / \eta+\eta k^{4}} .
$$

The contribution from diagram (a) then scales as

$$
\text { (a) } \propto \frac{1}{\eta} \int d \omega d^{3} k \frac{1}{\omega^{2} / \eta+\eta k^{4}} \sim \eta^{-5 / 4}
$$

while diagram (b) behaves as

$$
\text { (b) } \propto \int d \omega d^{3} k \frac{\omega^{2}}{\eta^{2}} \frac{1}{\left(\omega^{2} / \eta+\eta k^{4}\right)^{2}} \sim \eta^{-5 / 4},
$$

and we see that both vanish when we take $\eta \rightarrow \infty$. Therefore there are no new contributions at one loop from Higgs bosons, and the next to leading order calculation of $\sigma$ is unaffected. This will not be true in the case were there is a Higgs condensate of magnitude $\phi_{0} \sim$ $g T / \log (1 / g)$, because in that case the external Higgs field insertions on the $A$ field lines in the one loop diagrams considered in [12 will change the gauge field propagators by order 1 . The Higgs field will also be important beyond one loop, in the construction of an effective theory which can determine the $O(1 / \log )$ term in Eq. (1.3); but we will not investigate that problem here. 


\section{REFERENCES}

[1] G. t'Hooft, Phys. Rev. Lett. 37,8 (1976).

[2] V.A. Kuzmin, V.A. Rubakov and M.E. Shaposhnikov, Phys. Lett. B155, 36 (1985).

[3] P. Arnold and L. McLerran, Phys. Rev. D 36, 581 (1987).

[4] P. Arnold, D. Son and L.G. Yaffe, Phys. Rev. D55, 6264 (1997) [hep-ph/9609481].

[5] P. Huet and D.T. Son, Phys. Lett. B393, 94 (1997) [hep-ph/9610259].

[6] D.T. Son, UW/PT-97-19 [hep-ph/9707351].

[7] D. Bödeker, Phys. Lett. B 426, 351 (1998) [hep-ph/9801430].

[8] D. Bodeker, hep-ph/9905239; hep-ph/9903478.

[9] P. Arnold, D. T. Son and L. G. Yaffe, Phys. Rev. D59, 105020 (1999); Phys. Rev. D60, 025007 (1999) hep-ph/9901304.

[10] G. D. Moore, C. Hu and B. Muller, Phys. Rev. D58, 045001 (1998) hep-ph/9710436.

[11] D. Bodeker, G. D. Moore and K. Rummukainen, hep-ph/9907545.

[12] P. Arnold and L. G. Yaffe, hep-ph/9912306.

[13] P. Arnold and L. G. Yaffe, hep-ph/9912305.

[14] G.D. Moore, MCGILL/98-28 [hep-ph/9810313].

[15] P. Arnold, hep-ph/9912307.

[16] K. Kajantie, M. Laine, K. Rummukainen and M. Shaposhnikov, Nucl. Phys. B458, 90 (1996) hep-ph/9508379.

[17] K. Kajantie and J. Kapusta, Annals Phys. 160, 477 (1985).

[18] G. D. Moore, Phys. Rev. D59, 014503 (1999) hep-ph/9805264.

[19] K. Kajantie, M. Laine, K. Rummukainen and M. Shaposhnikov, Nucl. Phys. B493, 413 (1997) hep-lat/9612006.

[20] E. Braaten and R. Pisarski, Nucl. Phys. B337, 569 (1990);

[21] J. Frenkel and J. Taylor, Nucl. Phys. B334, 199 (1990); J. Taylor and S. Wong, Nucl. Phys. B346, 115 (1990); J. Frenkel and J. Taylor, Nucl. Phys. B 374, 156 (1992); E. Braaten and R. Pisarski, Phys. Rev. D 45, 1827 (1992).

[22] J.P. Blaizot and E. Iancu, Phys. Rev. Lett. 70, 3376 (1993) [hep-ph/9301236]; Nucl. Phys. B417, 608 (1994) hep-ph/9306294.

[23] V.P. Nair, Phys. Rev. D48, 3432 (1993) [hep-ph/9307326].

[24] E. Iancu, hep-ph/9710543.

[25] D. Bödeker, L. McLerran and A. Smilga, Phys. Rev. D52, 4675 (1995) [hep-th/9504123].

[26] G. Aarts, B. Nauta and C. G. van Weert, hep-ph/9911463.

[27] G. Aarts and J. Smit, Phys. Lett. B393, 395 (1997) [hep-ph/9610415].

[28] M. Laine, Nucl. Phys. B451, 484 (1995) hep-lat/9504001.

[29] M. Laine and A. Rajantie, Nucl. Phys. B513, 471 (1998) [hep-lat/9705003].

[30] K. Kajantie, M. Laine, K. Rummukainen and M. Shaposhnikov, Nucl. Phys. B466, 189 (1996) hep-lat/9510020.

[31] G. D. Moore and K. Rummukainen, hep-ph/9906259.

[32] G.D. Moore, Nucl. Phys. B523, 569 (1998) [hep-lat/9709053].

[33] J. Ambjørn and A. Krasnitz, Phys. Lett. B 362, 97 (1995) [hep-ph/9508202].

[34] K. Kajantie, M. Laine, K. Rummukainen and M. Shaposhnikov, Phys. Rev. Lett. 77, 2887 (1996) hep-ph/9605288].

[35] M. Thoma, Z. Phys. C 66, 491 (1995).

[36] U. Kraemmer, A. Rebhan, and H. Schulz, Ann. Phys. (N.Y.) 238, 286 (1995). 